\title{
Metodologias ativas no ensino de gestão em saúde no curso de medicina: o olhar discente
}

\author{
Active methodologies in the teaching of health management in the medical course: the \\ student's perspective
}

Metodologías activas en la enseñanza de la gestión de la salud en la carrera de medicina: la perspectiva del estudiante

Felipe Gabriel Silva Brito ${ }^{*}$, Antonia Iracilda e Silva Viana ${ }^{1}$, Bruna Pereira Carvalho Sirqueira ${ }^{1}$, Jacyane Ramos de Sousa ${ }^{2}$, Maricélia Tavares Borges Oliveira ${ }^{3}$, Danyelle Silva Martins ${ }^{4}$.

\section{RESUMO}

Objetivo: Analisar, a partir da perspectiva do discente o ensino da disciplina gestão em saúde através de metodologias ativas em um curso de medicina na região sul do Maranhão. Métodos: Estudo observacional, transversal e retrospectivo com 54 alunos do último ano do curso de medicina. Foi utilizado um questionário com perguntas agrupadas em domínios: objetivos da disciplina, metodologia utilizada, identificação de problemas no sistema de saúde, formação médica, estrutura física dos cenários de prática e autoavaliação do discente. Os dados obtidos foram analisados através do software $R$ ( $R$ Core Team, 2019) versão 3.5.3. $O$ estudo foi aprovado pelo Comitê de Ética em Pesquisa. Resultados: 83,3\% possuem de 20 a 29 anos; 55,6\% cor branca; $55,6 \%$ sexo feminino. Correlacionando-se a autoavaliação do aluno com aspectos da metodologia da disciplina houve significância estatística nos aspectos: objetivos da disciplina foram informados $(p<0,001)$, objetivos da disciplina foram alcançados ( $p<0,001)$, conteúdos da disciplina foram satisfatórios $(p=0,027)$, passou a conhecer um SUS que até então desconhecia $(p=0,0042)$. Conclusão: $A$ utilização de metodologias ativas proposta pelas mudanças nas diretrizes curriculares resultou em um processo de aprendizado mais dinâmico e crítico.

Palavras-chave: Avaliação educacional, Gestão em saúde, Educação médica.

\begin{abstract}
Objective: To analyze, from the student's perspective, the teaching of the discipline health management through active methodologies in a medical school in the southern region of Maranhão. Methods: Observational, cross-sectional and retrospective study with 54 final-year medical students. A questionnaire with questions grouped into domains was used: course objectives, methodology used, identification of problems in the health system, medical education, physical structure of practice scenarios and student self-assessment. The data obtained was analyzed using the $R$ software (R Core Team, 2019) version 3.5.3. The study was approved by the Research Ethics Committee. Results: $83.3 \%$ are between 20 and 29 years old; $55.6 \%$ white; $55.6 \%$ female. Correlating the student's self-assessment with aspects of the discipline's methodology, there was statistical significance in the aspects: discipline objectives were informed $(p<0.001)$, discipline objectives were achieved $(p<0.001)$, contents were satisfactory $(p=0.027)$, came to know SUS as they had not known before $(p=$ 0.0042). Conclusion: The use of active methodologies proposed by the changes in curriculum guidelines resulted in a learning process more dynamic and critical.
\end{abstract}

Keywords: Educational measurement, Health management, Medical education.

RESÚMEN

Objetivo: Analizar, desde la perspectiva del estudiante, la enseñanza de la disciplina de gestión de la salud a través de metodologías activas en un curso de medicina en la región sur de Maranhão. Métodos: Estudio observacional, transversal y retrospectivo con 54 estudiantes de último año de medicina. Se utilizó un

1 Universidade Federal do Maranhão (UFMA), Imperatriz - MA. *E-mail: felipe.silva.brito15@gmail.com

2 Escola de Saúde Pública do Estado do Maranhão (ESPMA), Imperatriz - MA.

${ }^{3}$ Universidade Federal do Tocantins (UFT), Palmas - TO.

${ }^{4}$ Universidade do Estado do Pará (UEPA), Belém - PA.

SUBMETIDO EM: 9/2021

ACEITO EM: 9/2021

PUBLICADO EM: 11/2021 
cuestionario con preguntas agrupadas en dominios: objetivos del curso, metodología utilizada, identificación de problemas en el sistema de salud, educación médica, estructura física de escenarios de práctica y autoevaluación del estudiante. Los datos obtenidos se analizaron mediante el software R (R Core Team, 2019) versión 3.5.3. El estudio fue aprobado por el Comité de Ética en Investigación. Resultados: El 83,3\% tiene entre 20 y 29 años; $55,6 \%$ de color blanco; $55,6 \%$ mujeres. Correlacionando la autoevaluación del estudiante con aspectos de la metodología de la disciplina, hubo significancia estadística en los aspectos: se informaron los objetivos de la disciplina ( $<<0,001)$, se alcanzaron los objetivos de la disciplina $(p<0,001)$, los contenidos de la disciplina fueron satisfactorios $(p=0,027)$, llegó a conocer un SUS que no conocía antes $(p=0,0042)$. Conclusión: El uso de metodologías activas propuestas por los cambios en los lineamientos curriculares resultó en un proceso de aprendizaje más dinámico y crítico.

Palabras clave: Evaluación educativa, Manejo de la salud, Educación médica.

\section{INTRODUÇÃO}

O modelo de medicina científica, também conhecido como modelo biomédico entra em crise na segunda metade do século 20, quando a ineficiência do mesmo se fez sentir por implicar em alto custo e pela ausência de resolubilidade às necessidades de saúde da população (RODRIGUES O, et al., 2013).

A partir da década de 60 movimentos mundiais questionavam acerca da formação médica que até então tinha como base o modelo Flexneriano, pautado em disciplinas isoladas que não se relacionavam entre si e repercutia em uma aprendizagem fragmentada. No Brasil a Associação Brasileira de Educação Médica integrou esse movimento em prol de mudanças na formação médica visando formar médicos generalistas que estivessem mais próximos da realidade social e que compreendesse a saúde de forma mais ampla (MACHADO CDB, et al., 2018).

A proposta de mudança do modelo pedagógico dos Cursos de Medicina visa, sobretudo, uma formação mais consoante com a prática médica, abandonando a estrutura flexineriana anterior que impõe um ensino centralizador, biologicista e com pouco diálogo com outras áreas do conhecimento humano, estabelecendo em seu lugar um modelo mais aberto centrado em metodologias ativas que estimulam a autonomia e o senso crítico dos discentes (FRANÇA JUNIOR RR e MAKNAMARA M, 2019).

Metodologias ativas consistem em formas de desenvolver o processo de aprendizagem utilizando experiências reais do cotidiano ou simuladas que podem representar situações da prática profissional em diferentes contextos com o objetivo de analisar determinado problema ou situação e encontrar soluções (BERBEL NN, 2011).

No Brasil, a Lei de Diretrizes e Bases para Educação Médica passou por profundas mudanças, promovidas pelos Ministérios da Educação e da Saúde baseadas principalmente nas Diretrizes Curriculares Nacionais para o Ensino de Graduação em Medicina (DCN). Programas como o de Incentivos a Mudança do Currículo Médico (PROMED), Programa Nacional de Reorientação da Formação Profissional em Saúde (Pró-Saúde) e Programa de Educação pelo Trabalho (PET-Saúde) foram implantados visando aproximar a formação médica do cenário dos serviços de saúde do SUS (BATISTA SHSS, et al., 2015).

A mudança da estrutura curricular se assemelha à uma perspectiva de "educação libertadora" como proposta por Paulo Freire, que intenta mobilizar social e politicamente os alunos para que eles possam adquirir uma postura de agentes de transformação da realidade em que estão inseridos (KOHAN WO, 2019).

De acordo com as Diretrizes Curriculares Nacionais (DCN) de 2014 os conteúdos curriculares que compõem a graduação médica devem estar orientados para as necessidades de saúde da população e fundamentados em uma concepção ampla de saúde que envolve aspectos referidos aos direitos humanos, ética, humanização, diversidade cultural entre outros. O contato do aluno desde o início da graduação com usuários e profissionais de saúde do SUS que atuam em diferentes serviços é um campo propício para conhecer os problemas reais de saúde vivenciados no cotidiano da Rede de Atenção à Saúde e aprimorar gradativamente o exercício do cuidado e o respeito ao ser humano em todas as suas dimensões (MINISTÉRIO DA EDUCAÇÃO, 2014). 
Os cursos de graduação em medicina têm implementado ambientes e módulos de aprendizagem que aproximem os discentes da realidade cotidiana da saúde, onde os problemas de saúde acontecem, como ocorrem e as variadas formas de intervenção. No contexto das Redes de Atenção à Saúde, destaca-se a gestão em saúde, que visa à formação do médico capaz de compreender a dinâmica e funcionamento do sistema de saúde, de forma a poder intervir em ações que possibilitem a tomada de decisões, orientadas para a solução de problemas decorrentes das necessidades de saúde da população e bem-estar da comunidade (COUTO VBM, et al., 2018).

A articulação entre o mundo da formação e o mundo do trabalho ocorre na medida em que o currículo se constitui por áreas de competência e de forma integrada. Nesse sentido o professor busca promover a aprendizagem reflexiva do estudante. Chirelli M e Nassif JV (2017) destacam capacidades centrais como o enfoque problematizador das vivências, que possibilita a reflexão e problematização a partir dos relatos dos estudantes sobre a perspectiva do que vivenciaram.

A preparação profissional durante a graduação para a atividade nos serviços de saúde é primordial para a atuação nos cenários de estágio estabelecidos no internato. Conhecimentos prévios com relação ao funcionamento dos serviços de saúde e os recursos disponíveis ao cuidado implicam uma menor carga de estresse e, por conseguinte uma melhor atuação (BOSCH J, et al., 2017). Na literatura pesquisada há farta publicação sobre metodologias ativas no ensino médico, porém em relação ao aspecto vivencial na Rede de Atenção à Saúde em diferentes níveis de atenção do SUS, as publicações são mais escassas (JÚNIOR CV, 2016; GOMES AP e REGO S, 2011; MOREIRA MB, et al., 2011; STENTOFT D, 2019).

O curso de graduação médica dos discentes pesquisados, tem como eixo curricular a metodologia Problem-Based Learning (PBL) centrada no aluno, como protagonista do processo de ensino-aprendizagem. Entretanto, não se constitui como única prática pedagógica, podendo o professor, sempre que julgar necessário e oportuno recorrer a outros métodos que se adequem a uma melhor compreensão da realidade que envolva a vida em sociedade e a prestação de serviços à comunidade como no caso da metodologia com enfoque de Problematização. Estudos apontaram fragilidades e potencialidades desse método, porém destacando a eficiência em sua aplicabilidade na formação médica com contribuições para um profissional proativo, resolutivo e com habilidades para o trabalho em equipe (SANTIAGO RC, et al., 2020).

Os conteúdos programáticos descritos na Ementa do Projeto Pedagógico do curso referente a Unidade modular Gestão em Saúde foram desenvolvidos em ambientes compatíveis com temas orientados para o novo perfil do profissional médico com atuação nos diferentes níveis da atenção à saúde visando a integralidade da assistência. Os ambientes em que se produzem saúde são relevantes para a prática médica. As DCN estabelecem que o processo de formação médica deve abranger o sistema de saúde brasileiro, a atenção integral à saúde, os processos de regionalização e fluxos de referência e contrarreferência (MINISTÉRIO DA EDUCAÇÃO, 2014).

Para contribuir sobre a importância das metodologias ativas na formação médica, o presente estudo objetivou analisar a partir da perspectiva e do olhar discente, a efetividade do modelo de ensino baseado em metodologias ativas no módulo de aprendizagem Gestão em Saúde no curso de medicina.

\section{MÉTODOS}

Trata-se de um estudo observacional, transversal e retrospectivo. A amostra escolhida para a realização da pesquisa foi composta pelos alunos do último ano do curso de medicina que correspondem aos $11^{\circ} \mathrm{e} 12^{\circ}$ períodos que antecedem ao internato somando um total de 60 alunos, sendo que 54 se dispuseram a responder à pesquisa $(n=54)$. O período elaboração e realização da pesquisa foi de outubro a dezembro de 2019.

A pesquisa utilizou como instrumento de coleta de dados a aplicação de questionários estruturados individuais disponibilizados em plataforma online Google Forms que possibilitaram ao acadêmico 05 níveis de resposta segundo a escala de Likert: 1- Discordo totalmente; 2 -Discordo parcialmente; 3 - Não concordo nem discordo; 4 - Concordo parcialmente; 5 - Concordo totalmente. Foi estipulado o prazo de no máximo 20 dias para o encerramento do preenchimento das respostas. 
As variáveis analisadas nos questionários contemplaram questões sobre: caracterização sociodemográfica; avaliação dos discentes sobre objetivos e conteúdo da disciplina; metodologia utilizada; conhecimento e funcionamento do sistema; contribuições da disciplina para formação médica e autoavaliação do aluno.

Os dados obtidos foram tabulados e codificados no programa Microsoft Excel e posteriormente exportados para análises no software R (R Core Team, 2019) versão 3.5.3. Os resultados foram descritos em tabelas de frequência absoluta e percentual. As associações entre as variáveis foram obtidas pelo teste Exato de Fisher. O nível de confiança adotado foi de 95\% e significância para valores de $p<0,05$.

A pesquisa foi aprovada no Comitê de Ética em Pesquisa com seres humanos da Universidade Federal do Maranhão (CAAE: 24680419.4.0000.5087) respeitando-se os princípios de autonomia, beneficência, não maleficência, justiça e equidade. Os participantes da pesquisa receberam e assinaram o Termo de Consentimento Livre e Esclarecido (TCLE) conforme a resolução 510/2016 do Conselho Nacional de Saúde (CNS).

\section{RESULTADOS}

Para caracterizar o perfil dos discentes, os dados foram mensurados através de frequências absolutas e relativas. O teste exato de Fisher foi utilizado para verificar a existência de associação entre a autoavaliação do discente, a metodologia utilizada, considerando o nível de significância de $5 \%$.

O estudo avaliou uma amostra de 54 estudantes $(n=54)$. A análise do perfil sociodemográfico da amostra estudada demonstrou que $45(83,3 \%)$ dos discentes estão dentro da faixa etária de 20 a 29 anos. Quanto à autodeclaração de etnia a maior prevalência é de indivíduos brancos no total de 30 (55,6\%). A renda familiar de maior parte da amostra se encontra entre 2 a 5 salários mínimos $(42,6 \%)$. A maioria dos estudantes não possui plano de saúde $(57,4 \%)$. As frequências relativas e absolutas das características sociodemográficas dos participantes da pesquisa estão descritas na Tabela 1.

Tabela 1 - Características sociodemográficas da amostra.

\begin{tabular}{lcc}
\hline Variáveis & $\mathbf{N}$ & $\%$ \\
\hline Sexo & 30 & 55,6 \\
\hline Feminino & 24 & 44,4 \\
Masculino & 45 & 83,3 \\
Faixa etária & 9 & 16,7 \\
20 a 29 anos & & \\
30 a 39 anos & 24 & 44,4 \\
\hline Período atual & 30 & 55,6 \\
\hline $11^{\circ}$ & & \\
$12^{\circ}$ & 30 & 55,6 \\
\hline Cor/Raça & 21 & 38,9 \\
\hline Branco & 03 & 5,5 \\
Pardo & & \\
Preta & 5 & 9,3 \\
Renda familiar & 23 & 42,6 \\
\hline a 2 salários-mínimos & 15 & 27,8 \\
5 a 10 salários-mínimos & 10 & 18,5 \\
Acima de 10 salários-mínimos & 1 & 1,9 \\
Até 1 salário-mínimo & & \\
\hline Você tem plano de saúde? & 31 & 57,4 \\
\hline Não & 23 & 42,6 \\
Sim & & \\
\hline Os membros da sua família possuem plano de saúde? & 25 & 46,3 \\
\hline Não & 29 & 53,7 \\
Sim &
\end{tabular}

Fonte: Brito FGS, et al., 2021. 
No que tange aos objetivos da disciplina a avaliação dos discentes demonstrou que $70,3 \%$ dos mesmos concordaram parcial ou totalmente que os objetivos da disciplina foram alcançados. Foi também demonstrado que $40,7 \%$ dos entrevistados concordaram totalmente que o conteúdo da disciplina de gestão em saúde referente ao Sistema Único de Saúde foi satisfatório e que os cenários de prática tinham estrutura física adequada. Concordaram parcialmente $27,8 \%$ em relação ao conteúdo e $29,6 \%$ em relação a adequação da estrutura física, conforme detalhamento na Tabela 2.

Tabela 2 - Avaliação dos discentes quanto aos objetivos e conteúdo da disciplina.

\begin{tabular}{lcc}
\hline Variáveis & $\mathbf{N}$ & $\%$ \\
\hline Os objetivos da disciplina foram alcançados & 4 & 7,4 \\
\hline Discordo totalmente & 4 & 7,4 \\
Discordo parcialmente & 8 & 14,8 \\
Não concordo, nem discordo & 20 & 37,0 \\
Concordo parcialmente & 18 & 33,3 \\
Concordo totalmente & & \\
\hline O conteúdo da disciplina de gestão em saúde referente ao Sistema Único de & & \\
Saúde foi satisfatório & 2 & 3,7 \\
\hline Discordo totalmente & 4 & 7,4 \\
Discordo parcialmente & 11 & 20,4 \\
Não concordo, nem discordo & 15 & 27,8 \\
Concordo parcialmente & 22 & 40,7 \\
Concordo totalmente & & \\
\hline Estrutura física dos cenários de prática foram adequados ao conteúdo & 4 & 7,4 \\
\hline Discordo parcialmente & 12 & 22,3 \\
Não concordo, nem discordo & 16 & 29,6 \\
Concordo parcialmente & 22 & 40,7 \\
Concordo totalmente & & \\
\hline Fonte: Brito FGS, et al., 2021.
\end{tabular}

Fonte: Brito FGS, et al., 2021.

Em relação a avaliação dos discentes sobre o conhecimento e funcionamento do SUS, verificou-se que houve criticidade em relação ao Sistema Único de Saúde especialmente no que trata do funcionamento e acesso do usuário aos serviços. Do total de sujeitos consultados na pesquisa $73,6 \%$ concordaram parcial ou totalmente que através do conhecimento adquirido no percurso das aulas de gestão em saúde passaram a conhecer um SUS que não sabiam que existiam. A maior parte dos alunos que responderam aos questionários $(74 \%)$ afirmaram total ou parcialmente que foi possível identificar os problemas reais que a população enfrenta no dia a dia no SUS.

Dentre os problemas identificados, $52,8 \%$ consideraram parcialmente que há dificuldade de acesso a medicamentos no SUS e 30,2\% concordaram totalmente que há dificuldade de acesso a medicamentos pelo SUS. Cerca de $88,4 \%$ concordaram total ou parcialmente que não há informações para os usuários sobre os serviços disponíveis no Sistema. Quase $85 \%$ dos entrevistados concordaram parcial ou totalmente que não houve continuidade no cuidado em tratamentos devido à falta de exames complementares para diagnóstico. Outro aspecto destacado pelos acadêmicos foi a falta de resolutividade da Atenção Básica apontado por $77,3 \%$ como um problema vivenciado no SUS que leva as pessoas a irem para Unidades de Pronto Atendimento ou Pronto Socorro Hospitalar, conforme Tabela 3. 
Tabela 3 - Avaliação dos discentes em relação ao conhecimento e funcionamento do SUS.

\begin{tabular}{|c|c|c|}
\hline Variáveis & $\mathbf{N}$ & $\%$ \\
\hline \multicolumn{3}{|c|}{ Passei a conhecer um SUS que não sabia que existia } \\
\hline Discordo totalmente & 3 & 5,7 \\
\hline Não concordo, nem discordo & 11 & 20,8 \\
\hline Concordo parcialmente & 26 & 49,1 \\
\hline Concordo totalmente & 13 & 24,5 \\
\hline \multicolumn{3}{|c|}{ O SUS precisa ser conhecido por todos os profissionais de saúde } \\
\hline Discordo totalmente & 0 & 0,0 \\
\hline Discordo parcialmente & 0 & 0,0 \\
\hline Não concordo, nem discordo & 7 & 13,2 \\
\hline Concordo parcialmente & 11 & 20,8 \\
\hline Concordo totalmente & 35 & 66,0 \\
\hline \multicolumn{3}{|c|}{$\begin{array}{l}\text { Foi possível identificar problemas reais que a população enfrenta no dia a dia } \\
\text { no SUS }\end{array}$} \\
\hline Discordo totalmente & 2 & 3,7 \\
\hline Discordo parcialmente & 4 & 7,4 \\
\hline Não concordo, nem discordo & 8 & 14,8 \\
\hline Concordo parcialmente & 16 & 29,6 \\
\hline Concordo totalmente & 24 & 44,4 \\
\hline \multicolumn{3}{|c|}{ Os pacientes têm dificuldade de acesso à medicamentos no SUS } \\
\hline Discordo totalmente & 0 & 0,0 \\
\hline Discordo parcialmente & 2 & 3,8 \\
\hline Não concordo, nem discordo & 7 & 13,2 \\
\hline Concordo parcialmente & 28 & 52,8 \\
\hline Concordo totalmente & 16 & 30,2 \\
\hline \multicolumn{3}{|c|}{ Falta de informação dos pacientes sobre os serviços disponíveis no SUS } \\
\hline Discordo totalmente & 0 & 0,0 \\
\hline Discordo parcialmente & 2 & 3,8 \\
\hline Não concordo, nem discordo & 4 & 7,7 \\
\hline Concordo parcialmente & 14 & 26,9 \\
\hline Concordo totalmente & 32 & 61,5 \\
\hline \multicolumn{3}{|c|}{ Falta de continuidade no cuidado por falta de exames diagnósticos } \\
\hline Discordo totalmente & 0 & 0,0 \\
\hline Discordo parcialmente & 2 & 3,8 \\
\hline Não concordo, nem discordo & 6 & 11,3 \\
\hline Concordo parcialmente & 21 & 39,6 \\
\hline Concordo totalmente & 24 & 45,3 \\
\hline \multicolumn{3}{|c|}{$\begin{array}{l}\text { Ausência de resolutividade nos postos de saúde faz com que o usuário } \\
\text { procure o Pronto Atendimento ou Pronto Socorro Hospitalar }\end{array}$} \\
\hline Discordo totalmente & 1 & 1,9 \\
\hline Discordo parcialmente & 7 & 13,2 \\
\hline Não concordo, nem discordo & 4 & 7,5 \\
\hline Concordo parcialmente & 14 & 26,4 \\
\hline Concordo totalmente & 27 & 50,9 \\
\hline
\end{tabular}

Fonte: Brito FGS, et al., 2021. 
As respostas às afirmativas sobre a avaliação que o acadêmico faz sobre o conhecimento adquirido no SUS e a formação médica evidenciaram que $87 \%$ dos alunos compreenderam que é importante conhecer o Sistema para poder atuar melhor nele e quase $90 \%$ que tal conhecimento pode contribuir para que possam orientar o paciente de forma adequada. Entre os que concordaram total ou parcialmente $81,5 \%$ reconhece que mesmo com limitações o SUS é muito abrangente e atende grande parte da população, conforme descrição na Tabela 4.

Tabela 4 - Avaliação dos discentes em torno da importância de conhecer o SUS para formação médica.

\begin{tabular}{lcc}
\hline Variáveis & N & $\%$ \\
\hline $\begin{array}{l}\text { Me fez compreender que o médico precisa conhecer como o sistema } \\
\text { funciona para atuar melhor dentro dele }\end{array}$ & & \\
\hline Discordo totalmente & 0 & 0 \\
Discordo parcialmente & 5 & 9,3 \\
Não concordo, nem discordo & 2 & 3,7 \\
Concordo parcialmente & 22 & 40,7 \\
Concordo totalmente & 25 & 46,3 \\
\hline Me possibilitou entender que o SUS é muito abrangente e atende a grandes & & \\
problemas de saúde da população & & 3,7 \\
\hline Discordo totalmente & 2 & 0 \\
Discordo parcialmente & 0 & 14,8 \\
Não concordo, nem discordo & 8 & 27,8 \\
Concordo parcialmente & 15 & 53,7 \\
Concordo totalmente & 29 & \\
\hline Contribuiu para que o médico saiba orientar o usuário no acesso ao serviço \\
de saúde
\end{tabular}

Fonte: Brito FGS, et al., 2021.

Em que medida a utilização de metodologias ativas possibilitou ao aluno uma autoavaliação em torno do seu aprendizado sobre o SUS foi demonstrado na Tabela 5. Houve significância estatística na correlação da autoavaliação com várias afirmativas relacionadas ao desenvolvimento da compreensão do sistema de saúde.

Nota-se que existiu uma associação entre a autoavaliação do discente e a percepção de significado e importância da disciplina ( $p<0,001)$; os objetivos informados da disciplina $(p<0,001)$; satisfação quanto ao conteúdo da disciplina ( $p=0,027)$; que a metodologia utilizada ajudou a conhecer um SUS que até então desconhecia $(p=0,042)$ conforme dados apresentados na Tabela 5. Nas demais variáveis estudadas não se observou uma associação significativa. 
Tabela 5 - Aspectos da metodologia associadas à autoavaliação do discente.

\begin{tabular}{|c|c|c|c|c|c|c|c|c|c|c|}
\hline \multirow{2}{*}{ Variáveis } & \multicolumn{2}{|c|}{ Ótimo } & \multicolumn{2}{|c|}{ Bom } & \multicolumn{3}{|c|}{ Regular } & \multicolumn{2}{|c|}{ Ruim } & \multirow{2}{*}{ p-valor } \\
\hline & $\mathbf{N}$ & $\%$ & $\mathbf{N}$ & $\%$ & $\mathbf{N}$ & & $\%$ & $\mathbf{N}$ & $\%$ & \\
\hline \multicolumn{10}{|c|}{ Ficou claro o significado e a importância da disciplina para o curso } & $<0,001$ \\
\hline Discordo totalmente & 0 & 0 & 1 & 3,2 & 0 & & 0 & 1 & 100 & \\
\hline Discordo parcialmente & 1 & 8,3 & 9 & 29 & 6 & & 60 & 0 & 0 & \\
\hline $\begin{array}{l}\text { Não concordo, nem } \\
\text { discordo }\end{array}$ & 1 & 8,3 & 9 & 29 & 1 & & 10 & 0 & 0 & \\
\hline Concordo totalmente & 10 & 83,3 & 12 & 38,7 & 3 & & 30 & 0 & 0 & \\
\hline \multicolumn{10}{|c|}{ Os objetivos da disciplina foram informados } & $<0,001$ \\
\hline Discordo totalmente & 0 & 0 & 1 & 3,2 & 0 & & 0,0 & 1 & 100,0 & \\
\hline Discordo parcialmente & 0 & 0 & 1 & 3,2 & 1 & & 10,0 & 0 & 0,0 & \\
\hline $\begin{array}{l}\text { Não concordo, nem } \\
\text { discordo }\end{array}$ & 1 & 8,3 & 11 & 35,5 & 2 & & 20,0 & 0 & 0,0 & \\
\hline Concordo parcialmente & 2 & 16,7 & 8 & 25,8 & 6 & & 60,0 & 0 & 0,0 & \\
\hline Concordo totalmente & 9 & 75,0 & 10 & 32,3 & 1 & & 10,0 & 0 & 0,0 & \\
\hline \multicolumn{10}{|c|}{ Os conteúdos da disciplina foram satisfatórios } & 0,027 \\
\hline Discordo totalmente & 0 & 0 & 0 & 0,0 & 2 & & 20,0 & 0 & 0,0 & \\
\hline Discordo parcialmente & 0 & 0 & 4 & 12,9 & 0 & & 0,0 & 0 & 0,0 & \\
\hline $\begin{array}{l}\text { Não concordo, nem } \\
\text { discordo }\end{array}$ & 0 & 0 & 6 & 19,4 & 4 & & 40,0 & 1 & 100,0 & \\
\hline Concordo parcialmente & 4 & 33,3 & 9 & 29,0 & 2 & & 20,0 & 0 & 0,0 & \\
\hline Concordo totalmente & 8 & 66,7 & 12 & 38,7 & 2 & & 20,0 & 0 & 0,0 & \\
\hline \multicolumn{10}{|c|}{ Achei que o SUS precisa ser conhecido por todos os profissionais de saúde } & 0,278 \\
\hline $\begin{array}{l}\text { Não concordo, nem } \\
\text { discordo }\end{array}$ & 0 & 0 & 5 & 16,7 & 1 & & 10 & 1 & 100 & \\
\hline Concordo parcialmente & 4 & 33,3 & 5 & 16,7 & 2 & & 20 & 0 & 0 & \\
\hline Concordo totalmente & 8 & 66,7 & 20 & 66,7 & 7 & & 70 & 0 & 0 & \\
\hline \multicolumn{10}{|c|}{ Me ajudou a conhecer o SUS que eu desconhecia } & 0,042 \\
\hline Discordo totalmente & & 0 & 0 & 4 & 12,9 & 1 & 10 & 1 & 100 & \\
\hline Discordo parcialmente & & 3 & 25 & 1 & 3,2 & 0 & 0 & 0 & 0 & \\
\hline Não concordo, nem discordo & & 0 & 0 & 5 & 16,1 & 1 & 10 & 0 & 0 & \\
\hline Concordo parcialmente & & 4 & 33,3 & 6 & 19,4 & 6 & 60 & 0 & 0 & \\
\hline Concordo totalmente & & 5 & 41,7 & 15 & 48,4 & 2 & 20 & 0 & 0 & \\
\hline \multicolumn{10}{|c|}{$\begin{array}{l}\text { Me fez compreender que o médico precisa conhecer como o sistema funciona para atuar } \\
\text { melhor dentro dele }\end{array}$} & 0,175 \\
\hline Discordo parcialmente & & 0 & 0 & 3 & 9,7 & 1 & 10 & 1 & 100 & \\
\hline Não concordo, nem discordo & & 2 & 16,7 & 0 & 0 & 0 & 0 & 0 & 0 & \\
\hline Concordo parcialmente & & 5 & 41,7 & 13 & 41,9 & 4 & 40 & 0 & 0 & \\
\hline Concordo totalmente & & 5 & 41,7 & 15 & 48,4 & 5 & 50 & 0 & 0 & \\
\hline \multicolumn{10}{|c|}{$\begin{array}{l}\text { Me possibilitou entender que o SUS é muito abrangente e atende a grandes problemas de } \\
\text { saúde da população }\end{array}$} & 0,474 \\
\hline Discordo totalmente & & 0 & 0 & 1 & 3,2 & 0 & 0 & 1 & 100 & \\
\hline Não concordo, nem discordo & & 1 & 8,3 & 5 & 16,1 & 2 & 20 & 0 & 0 & \\
\hline Concordo parcialmente & & 3 & 25 & 9 & 29 & 3 & 30 & 0 & 0 & \\
\hline Concordo totalmente & & 8 & 66,7 & 16 & 51,6 & 5 & 50 & 0 & 0 & \\
\hline
\end{tabular}

Fonte: Brito FGS, et al., 2021. 


\section{DISCUSSÃO}

O perfil sociodemográfico dos estudantes de medicina entrevistados demonstrou uma similaridade com outros estudos realizados com alunos da área da saúde em universidades federais com relação a: gênero, etnia, faixa etária e renda familiar média (ASSIS PYS, et al., 2015). A análise a partir da perspectiva de raça demonstrou um predomínio de pessoas que se autodeclararam como brancas $(55,6 \%)$ e um número ínfimo de acadêmicos que se identificam como negros (5,6\%).

Embora a Lei de Cotas 12.711/2012 prevê a reserva de vagas nas Instituições de Ensino Superior aos estudantes de escolas públicas de baixa renda, pretos, pardos e indígenas, esses resultados quando confrontados com os dados demográficos da população brasileira revelaram que as disparidades ao acesso à educação superior no Brasil persistem (BRASIL, 2012; ANDRADE CY, 2019). As políticas afirmativas e de inclusão que se realizaram por meio das cotas raciais buscou corrigir distorções e diminuir as desigualdades raciais e étnicas no acesso da população negra e outros grupos populacionais ao ensino superior (MARQUES EPS, 2018).

A avaliação dos sujeitos da pesquisa sobre o conhecimento e funcionamento do SUS apontou observações feitas pelos acadêmicos a partir da experiência com vários cenários que foi possível conhecer o SUS e identificar os problemas reais de saúde da população. Afirmações semelhantes são relatadas em estudo por Couto VBM, et al. (2018) sobre o ensino de gestão em saúde na graduação em medicina no que diz respeito aos entraves enfrentados pela gestão do sistema. Isto contribuiu para um melhor aproveitamento pelos acadêmicos, demonstrando que as mudanças pedagógicas no curso de Medicina conseguem alcançar seus objetivos estabelecidos, preparando o aluno não só com conhecimentos científicos sólidos, mas conhecimentos humanos e criticidade.

A criticidade ao sistema foi evidente na avaliação dos acadêmicos pesquisados, porém associada ao reconhecimento da importância do aprendizado teórico e inserção nos ambientes de prática. Resultados semelhantes foram relatados em estudos de Pereira GA (2018) sobre a concepção negativa de acadêmicos em relação ao SUS, mas que ao serem inseridos no cenário da Estratégia Saúde da Família logo no início do curso, essas concepções são descontruídas durante o processo de formação.

O acesso a medicamentos através do SUS foi incluído entre os problemas percebidos pelos acadêmicos. Os resultados mostraram que um total de $52,8 \%$ dos participantes concordou parcialmente e $30,2 \%$ concordaram totalmente que os pacientes têm dificuldades de acesso aos medicamentos. Embora o acesso a medicamentos seja assegurado no SUS pelo princípio da universalidade ele ocorre de forma desigual entre segmentos da população, atingindo especialmente os mais pobres, quando por vezes o usuário tem que fazer desembolso para obter os medicamentos necessários e garantir a continuidade do tratamento a que esteja submetido (OLIVEIRA LCF, et al., 2019). O acesso a medicamentos é um dos indicadores utilizados para mensurar os avanços na concretização do direito à saúde, porém apenas dois terços da população mundial possuem acesso regular a medicamentos (WORLD HEALTH ORGANIZATION, 2011).

Outra questão apontada foi a percepção quanto à resolutividade da atenção básica. Um total $77,3 \%$ dos acadêmicos entrevistados concordaram parcial ou totalmente que há uma falta de resolutividade na atenção básica que faz com que o usuário procure o pronto atendimento. Problemas relativos ao atendimento, disponibilidade de insumos e estrutura inadequada estão na base dessa falha (FACCHINI LA, et al., 2018). A falta de estrutura adequada das unidades públicas de saúde foi citada por Mendes TMC, et al. (2018) como um dos pontos que prejudica as atividades de ensino e serviço, em virtude de não oferecer espaço físico adequado para os profissionais, acadêmicos e usuários.

Em estudo realizado por Couto VBM, et al. (2018) sobre a experiência de graduandos de medicina na Rede de Atenção à Saúde constataram a mesma realidade apontada nessa pesquisa, a de que a Atenção Básica carece de resolutividade, não há fluxo de contrarreferência, com insuficiência de profissionais e ausência de medidas de acolhimento. Esses motivos fazem com que o usuário procure por Unidades onde realizam consultas médicas e tenham especialistas, ou mesmo as Unidades de Pronto Atendimento ou Hospitais. 
O modelo de ensino vigente que utiliza metodologias ativas coloca o estudante como protagonista do seu aprendizado, tendo como elemento fundamental sua autonomia e gerando um processo reflexivo e de problematização da realidade para que o mesmo consiga elaborar estratégias inovadoras para a solução de problemas (DIESEL A, et al., 2017). Resultados semelhantes de avaliação positiva da integração com os serviços de saúde e comunidade foram encontrados em estudos que apontaram o contato com a comunidade, como um meio que além de possibilitar a aproximação com a realidade sanitária e social do país, permite visualizar o cerne de muitos problemas de saúde (MENDES TMC, et al., 2020).

A análise de boas avaliações pessoais feitas pelos discentes, visando alcançar os objetivos estabelecidos pelo módulo, reforça os princípios do ensino fundamentado nas metodologias ativas em que o aluno se torna capaz de desenvolver a criticidade, reflexão e tomada de decisão. Ao avaliar a abrangência do SUS e suas limitações, apesar dos entraves que foram observados, compreenderam que o sistema consegue beneficiar a maioria da população.

Avaliações positivas em relação ao Sistema Único de Saúde foram encontradas em estudos de Araújo LPG, et al. (2018) onde estudantes constataram que apesar das condições precárias de atendimento, o sistema atende milhares de pessoas que não teriam outra forma de acesso em virtude dos altos custos dos planos de saúde. Embora o estudo apresente percepções negativas por parte de alunos sobre o SUS, traz observações de que a percepção do discente sobre o sistema vai depender em parte da experiência vivenciada durante a graduação e das etapas e períodos que estiver cursando.

Desse modo, a gestão em saúde é um aspecto da dimensão do SUS organizado por níveis de atenção, que demanda complexidade, porém o seu conhecimento auxilia na tomada de decisões, na percepção sobre a funcionalidade do sistema, formulações de políticas micro e macro setorial que possam promover uma ideia ampliada dos possíveis pontos da gestão que devem ser observados e modificados de forma a atender as necessidades de saúde da comunidade (CANTO IM e ALMEIDA MJ, 2019).

\section{CONCLUSÃO}

A experiência com metodologias ativas no ensino de gestão em saúde foi considerada pelos alunos do internato como necessária e importante por possibilitar que o futuro médico conheça o sistema de saúde para atuar melhor nele. Compreenderam que o sistema é abrangente e tem entraves, como por exemplo a falta de continuidade em tratamentos por falta de exames e medicamentos, porém é imprescindível para assistência à saúde da maioria da população brasileira. Com base nos resultados pode-se inferir que a inserção dos alunos na convivência com usuários, trabalhadores de saúde através de metodologias ativas pode contribuir para a formação de médicos mais humanos, éticos e comprometidos com a melhoria da situação de saúde da população.

\section{REFERÊNCIAS}

1. ANDRADE CY. Jovens, raça e renda: o alcance limitado das políticas de inclusão e a permanência das desigualdades educacionais. Anais, 2019; 1-18 p.

2. ARAUJO LPG, et al. O SUS que ninguém vê: experiência de estudantes de medicina inseridos em uma comunidade do município de Itaperuna/RJ. Revista Interdisciplinar do Pensamento Científico, 2018; 4(1): 42-50.

3. ASSIS PYS, et al. Características sociodemográficas e acadêmicas dos discentes da área da saúde. Revista da Universidade Vale do Rio Verde, 2015; 13(1):154-164.

4. BRASIL. Presidência da República. Casa Civil. Lei $n^{\circ} 12.711$ de 29 de agosto de 2012. Dispõe sobre o ingresso nas universidades federais e nas instituições federais de ensino técnico de nível médio e dá outras providências. Disponível em http://www.planalto.gov.br/ccivil_03/_ato2011-2014/2012/lei/l12711.htm. Acesso em 19.10.2021.

5. BATISTA SHSS, et al. Formação em Saúde: reflexões a partir dos programas Pró - Saúde Interface 2015; 19(supp 1).

6. BERBEL NN. As metodologias ativas e a promoção da autonomia dos estudantes. Semina: Ciências Sociais e Humanas, 2011; 32(1): 25-40.

7. $\mathrm{BOSCH}$ J, et al. Medical students' preparedness for professional activities in early clerkships. BMC medical education, 2017;17(1): 140.

8. CANTO IM, ALMEIDA MJ. O Ensino da Gestão em Saúde nos cursos de Enfermagem e Medicina. Espaço para Saúde. 2019; 20(1): 62-64. 
9. CHIRELLI M, NASSIF JV. Metodologia Ativa no currículo por competência: processo, facilidades e dificuldades. Atas CIAIQ, 2017: Investigacão Qualitativa em Educação, 2017; 1: 518-529.

10. COUTO VBM, et al. Vivenciando a Rede: Caminhos para a formação do médico no contexto do SUS. Revista Brasileira de Educação Médica. 2018; 42(2): 5-14.

11. DIESEL A, et al. Os princípios das metodologias ativas de ensino: uma abordagem teórica. Revista Thema, 2017; 14(1): 268-288.

12. FACCHINI LA, et al. Qualidade da atenção Primaria a Saúde no Brasil: Saúde debate. 2018; 42(sep 1).

13. FRANÇA JUNIOR RR, MAKNAMARA M. A literatura sobre metodologias ativas em educação médica no brasil: notas para uma reflexão crítica. Trabalho, Educação e Saúde, 2019; 17(1): 1-22.

14. GOMES AP, REGO S. Transformação da educação médica: é possível formar um novo médico a partir de mudanças no método de ensino-aprendizagem. Rev bras educ méd., 2011; 35(4): 557-66.

15. JÚNIOR CV. Metodologia ativa na educação médica. Rev Med (São Paulo). 2016; 95(3): 113-124.

16. KOHAN WO. Paulo Freire e o valor da igualdade em educação. Educação e Pesquisa, $2019 ; 45$.

17. MACHADO CDB, Educação Médica no Brasil: uma análise histórica sobre a formação acadêmica e pedagógica. Rev. bras. educ. med, 2018; 42(4).

18. MARQUES EPS. O acesso à educação superior e o fortalecimento da identidade negra. Revista Brasileira de Educação, 2018; 23: e230098

19. MENDES TMC, et al. Integração ensino-serviço-comunidade no Brasil e o que dizem os autores dos cenários de prática: uma revisão integrativa. Revista Ciência Plural, 2018; 4(1):98-116.

20. MENDES TMC, et al. Contribuições e desafios da integração ensino-serviço-comunidade. Texto \& ContextoEnfermagem, 2020; 29.

21. MINISTÉRIO DA EDUCAÇÃO. Conselho Nacional de Educação. Câmara de Educação Superior. Resolução $n^{\circ} 3$, DE 20 de junho de 2014, Institui Diretrizes Curriculares Nacionais do Curso de Graduação em Medicina e dá outras providências. Resolução CNE/CES 3/2014. Diário Oficial da União. Brasília-DF, Brasil, 23 de junho de 2014 - Seção 1 - pp. 8-11. Disponível em http://portal.mec.gov.br/index.php?option=com_docman\&view=download\&alias=15874rces003-14\&category_slug=junho-2014-pdf\&ltemid=30192. Acesso em 19.10. 2021.

22. MOREIRA MB, et al. O papel da aprendizagem baseada em problemas nas mudanças no ensino médico no Brasil. Rev HCPA, 2011; 31(4)

23. OLIVEIRA LCF, et al. O acesso a Medicamentos em sistemas universas em Saúde- Perspectivas e desafios. Saúde debate, 2019; 43 (spe 5).

24. PEREIRA, et al. O olhar do estudante de medicina sobre o Sistema Único de Saúde: a inflência de sua formação. Revista brasileira de educação médica, 2018; 42(3): 57-66.

25. RODRIGUES O, et al. Diretrizes Curriculares para o curso de Medicina- uma década depois. Vittalle, Rio Grande, 2013; 25(1): 29-35.

26. SANTIAGO RC, et al. Percepção dos estudantes de medicina sobre o uso da metodologia da problematização durante a graduação. Revista Brasileira de educação medica, 2020; 44(4):161

27. STENTOFT D. Problem-based projects in medical education: extending PBL practices and broadening learning perspectives. Adv Health Sci Educ Theory Pract, 2019; 24(5): 959-969.

28. WORLD HEALTH ORGANIZATION (WHO). The world medicines situation. Access to essential medicines as part of the right to health. Geneva: World Health Organization; 2011. Disponível em: https://apps.who.int/iris/handle/10665/78334 acesso em: 03.09.2021. 